\title{
Generation of Mycobacterium tuberculosis-specific recombinant antigens and evaluation of the clinical value of antibody detection for serological diagnosis of pulmonary tuberculosis
}

\author{
XIA ZHANG ${ }^{1,2^{*}}$, ZHONGLAN SU ${ }^{3 *}$, XIANGRONG ZHANG ${ }^{2}$, CHUNMEI HU ${ }^{2}$, \\ JUN YU ${ }^{2}$, QIAN GAO ${ }^{1}$ and HONGWEI WANG ${ }^{1}$ \\ ${ }^{1}$ Center for Translational Medicine and Jiangsu Key Laboratory of Molecular Medicine, Medical School of Nanjing University, \\ Nanjing, Jiangsu 210093; ${ }^{2}$ Department of Tuberculosis, Nanjing Chest Hospital, Nanjing; ${ }^{3}$ Department of Dermatology, \\ The First Affiliated Hospital of Nanjing Medical University, Nanjing, Jiangsu 210029, P.R. China
}

Received October 31, 2012; Accepted December 14, 2012

DOI: $10.3892 /$ ijmm.2013.1254

\begin{abstract}
Rapid diagnosis of pulmonary tuberculosis (TB) infection is critical in clinical practice. To establish an effective serological diagnostic technique, we generated the several Mycobacterium tuberculosis (MTB)-specific immunogenic antigens and evaluated the clinical benefits of detection of immunoglobulin $\mathrm{G}(\mathrm{IgG})$ and $\operatorname{IgM}$ antibodies raised against these target antigens for the diagnosis of patients with active TB. The genes encoding the MTB-specific antigens 6-kDa early secretory antigenic target of MTB (ESAT-6), 10-kDa culture filtrate protein (CFP-10), ESX-1 substrate protein C (ESPC), 14KD/38KD and ESAT-6/14KD/38KD, were amplified from the MTB genome by PCR. Prokaryotic vectors were constructed for the expression of the individual MTB antigens. The target recombinant protein was expressed in Escherichia coli (BL21/DE3) and purified using immobilized metal affinity chromatography (IMAC). An ELISA based immunoassay was set up using these target antigens for the diagnosis of active TB. The detection samples included 98 patients with active TB and 102 healthy control volunteers. The cutoff OD value for IgG and IgM antibodies was selected according to a receiver operating characteristic (ROC) analysis. The sensitivity, specificity and positive likelihood ratio were also determined. We successfully cloned, expressed and purified the ESAT-6, CFP-10, ESPC, 14KD/38KD and ESAT-6/14KD/38KD recombinant antigens of MTB. The
\end{abstract}

Correspondence to: Dr Hongwei Wang or Professor Qian Gao, Center for Translational Medicine and Jiangsu Key Laboratory of Molecular Medicine, 22 Hankou Road, Medical School of Nanjing University, Nanjing, Jiangsu 210093, P.R. China

E-mail: hwang@nju.edu.cn

E-mail: qian_gao@nju.edu.cn

${ }^{*}$ Contributed equally

Key words: pulmonary tuberculosis, serodiagnosis, BCG, recombinant antigen mean levels of IgG antibodies were significantly higher in patients with pulmonary TB compared with control groups. The target MTB-specific antigens can distinguish a TB infection from a non-TB infection, showing significant difference in statistics $(\mathrm{P}<0.001)$. The sensitivity of the $\mathrm{IgG}$ test ranged from $69.4 \%$ (ESAT-6) to $77.6 \%$ (ESAT-6/14KD/38KD) in the active TB patients; the specificity of assays varied from $78.4 \%$ (CFP-10) to $90.2 \%(14 \mathrm{KD}+38 \mathrm{KD})$ in the healthy control groups. The IgM antibody test can not distinguish a TB infection from a non-TB healthy control. In conclusion, clinical use of the ESAT-6, CFP-10, ESPC, 14KD/38KD and ESAT-6/14KD/38KD antigens based on serodiagnostic IgG assay is of significant value for the rapid diagnosis of TB and for the discrimination between active TB patients and healthy controls.

\section{Introduction}

Mycobacterium tuberculosis (MTB) is a leading cause of morbidity and mortality worldwide. It is estimated that 1.6 million people succumb to tuberculosis (TB) infection and nine million new cases of active TB are reported each year (1). One third of the world's population lives with TB. In developing countries, TB infection remains a major health problem.

Elimination of TB largely depends on definitive rapid diagnosis and treatment. A rapid, simple and relatively inexpensive diagnostic test is crucial for future control efforts. The most common laboratory-based detection techniques used to identify MTB are mycobacterial culture and acid-fast bacillus (AFB) staining (2). Although mycobacterial culture continues to be a valuable diagnostic tool, it usually requires several weeks to obtain the results. AFB staining provides rapid evidence for the presence of mycobacteria in a clinical specimen, however, $40-60 \%$ of patients with pulmonary TB infection and approximately $75 \%$ of patients with extrapulmonary TB infection are smear negative (3).

Immunological detection methods provide a useful approach for the diagnosis of TB. The major advantages of immunological detection are speed and simplicity, and it is 
also useful for the detection of extra-pulmonary TB or smear negative TB. Although considerable efforts have been made for the development of immune-based tests for the detection of antibodies, antigens, and immune complexes, their performance varies widely. Therefore, the development of rapid and accurate new diagnostic tools is crucial. Antibody detection is one of the most convenient forms for the TB immunodiagnostic test, which relies on the MTB-specific antigen. Despite extensive studies on mycobacterial antigens over the past 100 years, so far only a few serodiagnostic kits are available. Due to the common antigen existing in MTB and M. bovis Bacillus Calmette-Guérin (BCG), selection of MTB-specific antigens for antibody detection is key in distinguishing the MTB infection or BCG vaccination.

In the present study, we cloned, expressed and purified several MTB recombinant proteins including 6-kDa early secretory antigenic target of MTB (ESAT-6), 10-kDa culture filtrate protein (CFP-10), ESX-1 substrate protein C (ESPC), $14 \mathrm{KD} / 38 \mathrm{KD}$, and ESAT-6/14KD/38KD antigens. Based on these antigens, we also set up the ELISA-based serological test and evaluated the immunoreactivity of the target proteins with sera from pulmonary TB patients and healthy controls. We demonstrated that the serological detection of $\mathrm{IgG}$ antibody against the target MTB antigens can successfully distinguish the active TB-infected patient from the healthy control. This study may help identify the MTB-specific antigens to improve the serological detection sensitivity for MTB.

\section{Materials and methods}

Study population and serum sample collection. A total of 98 serum samples were obtained from patients (65 males and 33 females, aged between 16 to 84 years) suspected to have active pulmonary TB. All were patients at Nanjing Chest Hospital and had not received any anti-TB chemotherapy when the serum samples were collected. No patient identified as having the HIV-1 infection was included in this study. All subjects provided informed consent to participate in this study. The study population of TB patients was divided into the following groups: Group I included the mycobacterium positive pulmonary TB patients $(\mathrm{n}=54)$; Group II included the mycobacterium negative pulmonary TB patients $(n=44)$; Group III was the healthy individuals of this locality, which included 102 healthy control volunteers who had reported previously receiving BCG vaccination. The TB patients and healthy control volunteers were confirmed with the following methods: X-ray, clinical symptoms and patient history. Blood samples were collected, sera were separated, and serum specimens were obtained upon admission prior to any therapy and stored at $-80^{\circ} \mathrm{C}$ until testing.

Cloning, expression and purification of recombinant MTB antigens. Related gene encoding individual MTB antigen was amplified from H37RV genomic DNA by using the codon-optimized primers (Table I). The PCR product was sub-cloned into the vector pET-30a and pET-32a (Novagen) using the relevant restriction enzyme recognition sites. An His6-tag fusion peptide was attached at the C-terminus of the MTB protein, and a Trx.Tag was fusion expressed on the $\mathrm{N}$-terminus of the MTB ESAT-6, CFP-10 and ESPC antigens.

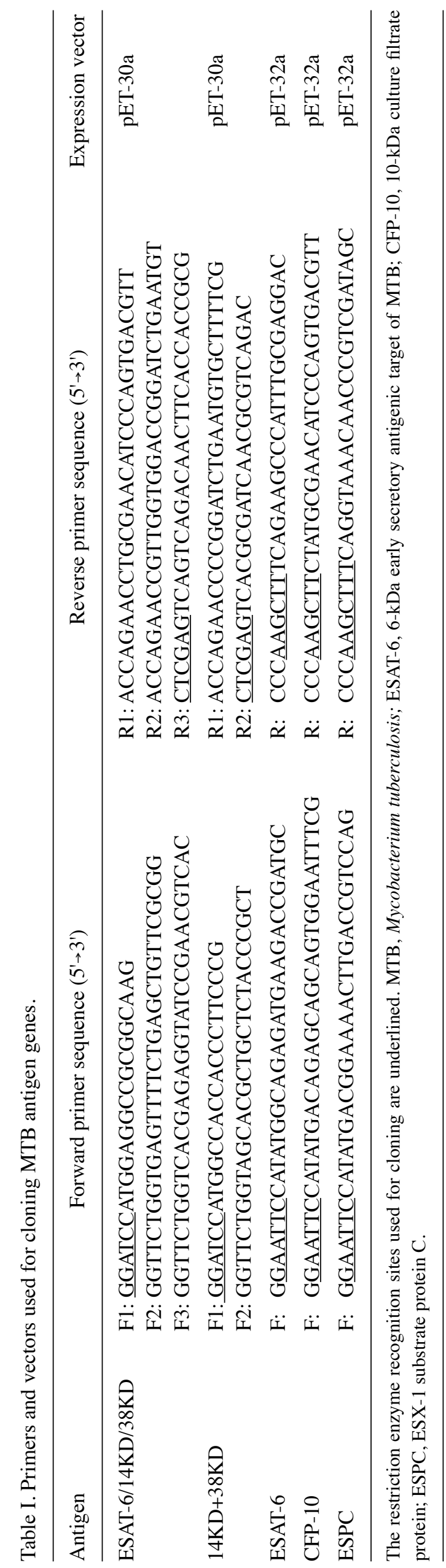


The correctness of the sequence was confirmed by DNA sequencing analysis.

For the production of recombinant protein, the obtained construct pET-30-MTB and pET-32-MTB coded for the target recombinant $\mathrm{MTB}$ proteins was transformed into Escherichia coli (E. coli) BL21(DE3) Rosetta 2 (Novagen) cells. The recombinant cells were grown in 4 liters of $2 \mathrm{YT}$ media at $37^{\circ} \mathrm{C}$, shaken at $110 \mathrm{rpm}$ in chicane flasks and induced with $2 \mathrm{mM}$ isopropyl- $\beta$-D-thiogalactopyranoside (IPTG) at an optical density (OD) 6001.5 for $4 \mathrm{~h}$ at $37^{\circ} \mathrm{C}$. Subsequently, the cells were harvested by a 10 -min centrifugation at $6,000 \mathrm{x} \mathrm{g}$, and were re-suspended in TBS buffer (containing $20 \mathrm{mM}$ HEPES pH 7.5).

To purify the recombinant MTB protein, the lysate supernatant was applied to a $10 \mathrm{ml} \mathrm{Ni-NTA} \mathrm{(Qiagen)} \mathrm{column}$ equilibrated with TBS, washed with 10 column volumes (CV) of TBS, and eluted with $2 \mathrm{CV}$ of $500 \mathrm{mM}$ imidazole in TBS. The eluted protein was concentrated to $5 \mathrm{ml}$ with a centrifugal filter concentration apparatus $(10 \mathrm{kDa}$ molecular mass cutoff, cellulose membrane; Millipore) and further purified by size-exclusion chromatography (Sephacryl S-100 HiPrep 26/60, TBS equilibrated) using an AKTAprime system (GE Healthcare) at a flow-rate of $1.0 \mathrm{ml} / \mathrm{min}$. Fractions containing the purified protein of interest were concentrated as before to $10 \mathrm{mg} / \mathrm{ml}$. The final protein concentration was measured using a bicinchoninic acid (BCA) kit (Pierce) and a spectrophotometer. The protein purity was confirmed by the presence of a single band on $12 \%$ sodium dodecyl-sulphate polyacrylamide gel electrophoresis (SDS-PAGE) stained with Coomassie Brilliant Blue.

Antibody detection by ELISA. The ELISA procedure was performed as follows: 96 -well polystyrene microtiter plates were coated overnight at $4^{\circ} \mathrm{C}$ with $100 \mu$ l of antigen solution ( $1 \mu \mathrm{g} / \mathrm{ml}$ for final concentration) in phosphate-buffered saline (PBS, pH 7.4). The coated plates were washed twice with PBS-T (PBS; $0.05 \%$ Tween-20, pH 7.4), and blocked with $1 \%$ bovine serum albumin (BSA) in PBS-T for $2 \mathrm{~h}$ at room temperature. Serum dilution of 1:200 was used in the assay. Serum samples were added and incubated for $2 \mathrm{~h}$ at room temperature. The plates were washed three times with PBS-T and then incubated for $1 \mathrm{~h}$ with rabbit anti-human IgG or rabbit antihuman IgM antibody conjugated with horseradish peroxidase diluted 1:10,000 in PBS-T. After five washes with PBS-T, the enzyme activity was assayed by incubation for $15 \mathrm{~min}$ at room temperature with $100 \mu \mathrm{l}$ of mixtures of tetramethylbenzidine (TMB) $(10 \mu \mathrm{g}$ TMB) per well. Then, $50 \mu \mathrm{l}$ of $1 \mathrm{~N}$ sulfuric acid was added to stop the reaction and the OD was evaluated in $450 \mathrm{~nm}$. The serum samples were tested in duplicate wells and at least three repeat experiments were performed to verify the reproducibility of results.

Data management and statistical analysis. The statistical analysis was performed as previously described (4). Briefly, the means \pm standard deviation of the OD of individual groups were determined in comparison with the mean OD value for the healthy control serum samples \pm standard deviations. The ROC curve and cutoff value was calculated using the MedCalc statistical software (Broekstraat, Belgium). The individual samples were scored as positive for the specific antibody

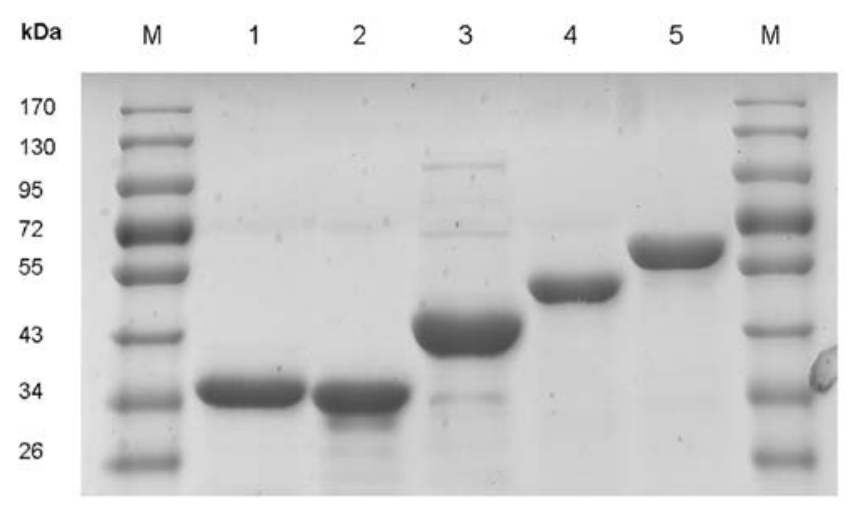

Figure 1. SDS-PAGE analysis of purified MTB recombinant proteins Lane 1, molecular weight markers; lane 2, purified ESAT-6 proteins; lane 3, purified CFP-10 proteins; lane 4, purified ESPC proteins; lane 5, purified 14KD/38KD proteins; lane 6, purified ESAT-6/14KD/38KD proteins. Molecular weight is kDa respectively. Proteins were visualized with Coomassie Brilliant Blue staining.

response when the OD value was above the cutoff value. Sensitivity was determined by dividing the number of positive cases by the total number of TB patients. Specificity was determined by dividing the number of negative controls by the total number of healthy controls. The differences among groups were analyzed by the Mann-Whitney test using the SPSS for Windows statistical software (SPSS Inc., Chicago, IL, USA). Differences were considered statistically significant at $\mathrm{P}<0.05$.

\section{Results}

Generation of MTB recombinant antigens. To generate the MTB recombinant antigens for immunoassay, we first amplified the individual MTB antigen encoding gene by PCR. Five MTB related antigen genes including the gene encoding CFP-10, ESAT-6, ESPC antigen, ESAT-6/14KD/38KD fusion protein, $14 \mathrm{KD} / 38 \mathrm{KD}$ fusion protein, were successfully constructed. The corresponding genes were inserted into the individual pET vectors using either BamHI or XhoI for pET-30a vector, or using EcoRI and HindIII restriction site for $\mathrm{pET}-32 \mathrm{a}$ vector. The construction of recombinant plasmids which expressed CFP-10, ESAT-6 and ESPC antigen protein were fusion expressed with an N-terminal Trx.Tag and a C-terminal His6-tag, which were confirmed by DNA sequencing. The expression of the corresponding MTB recombinant proteins was induced by IPTG in E. Coli BL21(DE3). The expression product was further purified by metal chelate affinity chromatography (Fig. 1); $>90 \%$ purity was achieved, measured by SDS-PAGE assay.

Serological test of active TB infection using recombinant antigens. We generated and identified the serodiagnostic potential of various MTB-specific antigens including ESAT-6, CFP-10, ESPC, ESAT-6/14KD/38KD and 14KD/38KD recombinant proteins. Using ELISA, we characterized the humoral immune response during TB infection by measuring serum IgG and IgM antibodies to mycobacterial antigen in 98 patients with active TB compared to 102 healthy control. 
Table II. IgG reactivity against various MTB antigens.

\begin{tabular}{lcccccccc}
\hline Antigen & Cutoff & Sensitivity (\%) & Specificity $(\%)$ & PPV & NPV & +LR & -LR & ROC area \\
\hline ESAT-6/14KD/38KD & 0.365 & 77.6 & 84.3 & 82.6 & 79.6 & 4.94 & 0.27 & 0.867 \\
14+38 kDa & 0.378 & 74.5 & 90.2 & 88.1 & 78.6 & 7.6 & 0.28 & 0.873 \\
ESAT-6 & 0.268 & 69.4 & 89.2 & 86.1 & 75.2 & 6.43 & 0.34 & 0.842 \\
CFP-10 & 0.241 & 72.5 & 78.4 & 76.3 & 74.8 & 3.36 & 0.35 & 0.804 \\
ESPC & 0.245 & 75.5 & 84.3 & 82.2 & 78.2 & 4.81 & 0.29 & 0.855 \\
\hline
\end{tabular}

IgG, immunoglobulin G; MTB, Mycobacterium tuberculosis; PPV, positive predictive value; NPV, negative predictive value; LR, logistic regression analysis; ROC, receiver operating characteristic; ESAT-6, 6-kDa early secretory antigenic target of MTB; CFP-10, 10-kDa culture filtrate protein; ESPC, ESX-1 substrate protein C.

Mean \pm SD values of $\operatorname{IgG}$ and $\operatorname{IgM}$ antibody titers to the individual mycobacterial antigens in active TB patients were determined. Sensitivity and specificity for a particular antigen were determined using ROC analysis. For all the antigens, the cutoff was selected at the point which showed the best accuracy, sensitivity and specificity by ROC. A predictive value (PV) to define the probability of a disease was also analyzed and included the positive predictive value (PPV) to characterize a patient for the particular disease from the patient's population, and a negative predictive value (NPV) to exclude the disease. The OD value of TB patients and control subjects with $\mathrm{IgG}$ antibodies to the individual mycobacterial antigen are summarized in Table II. The areas of under the curves and 95\% CI were also calculated (Table II). The OD value of IgM antibody detection results is not shown, as there were no significant differences between the healthy control subjects and active TB patients.

We found the levels of IgG antibodies against each antigen in TB patients were significantly higher than those that in the healthy controls $(\mathrm{P}<0.01)$ (Fig. 2). However, regarding the IgM antibody detection, we found that the IgM level did not differ between the examined active TB and the healthy control group; a relatively low OD value was obtained in both assays (Fig. 2). Therefore, this result indicated that characterization of IgG antibodies against these antigens can effectively distinguish between TB patients and healthy controls.

We observed the $\operatorname{IgG}$ antibody reactivity to the individual antigens, ESAT-6, CFP-10, ESPC, ESAT-6/14KD/38KD and $14 \mathrm{KD} / 38 \mathrm{KD}$ components was $69.4,72.5,75.5,77.6$ and $74.5 \%$; specificity was $89.2,78.4,84.3,84.3$ and $90.2 \%$, respectively. The overall positive rates of the detection of MTB IgG antibodies against each recombinant antigen by ELISA were calculated. The positive rates in active TB patients were significantly higher than those in healthy controls $(\mathrm{P}<0.01)$. Using the cutoff established by ROC, we determined that the positive rate for ESAT-6, CFP-10, ESPC, 14KD/38KD and ESAT-6/14KD/38KD antigens was $65,73,77,80$ and $82 \%$, respectively. We noted that the positive rates for antibody responses to multiple antigens such as ESAT-6/14KD/38KD and $14 \mathrm{KD} / 38 \mathrm{KD}$ antigens were slightly increased and at the same time the specificity was at approximately the comparable level. Therefore, detection of serum antibody responses to multiple antigens may be valuable for the diagnosis of MTB infection. Moreover, we did not observe any difference regarding to the detection rate, the sensitivity and specificity by comparing the mycobacterium positive and negative group (data not shown).

\section{Discussion}

Currently, the test used for the diagnosis of MTB infection includes sputum examination for the presence of MTB, culture of sputum or other body fluid, the tuberculin skin test and radiology, which is either insensitive or time consuming. In search of rapid and cost-effective diagnostic methods for MTB infection, immunodiagnosis, which uses the specific humoral and cellular immune responses of the host to identify the presence of infection or disease, is considered an attractive option. During the MTB infection, although the cellular immune responses are more important than the humoral immune responses in mediated immune protection, the antibody-mediated immune response can be used to set up serodiagnosis of MTB infection (5). MTB serodiagnosis is considerably simpler and less expensive and it has additional advantages in situations when the patient is unable to produce adequate sputum or when sputum smear results are negative and TB is extra-pulmonary.

For the establishment of effective immunoassays, extensive studies have been carried out for the identification of MTB-specific immunodominant antigens (6). Purification of these immunogenic antigens directly from MTB is challenging due to low cell yields, slow growth rates and the virulent nature of the bacteria (7). A potential solution to this problem is the production of recombinant antigens in organisms such as E.coli. In this study, we successfully cloned, expressed and purified several MTB antigens including ESAT-6, CFP-10, ESPC, ESAT-6/14KD/38KD, 14KD/38KD recombinant proteins and evaluated their diagnostic potential by ELISA for the detection of MTB-specific antibodies. By comparing the healthy individuals with confirmed TB patients, we found higher levels of MTB-specific IgG antibodies can be detected in the sera of TB patients. The levels of MTB antigen-specific IgG antibodies in TB patients are much higher than those in healthy controls, indicating that serological tests for MTB IgG antibodies against these MTB antigens are beneficial for the diagnosis of MTB infection.

The antigens selected for the current study have been reported to be strong targets for humoral and cell-mediated immune responses. These target antigen genes are conserved 
ESAT-6 $\lg G$

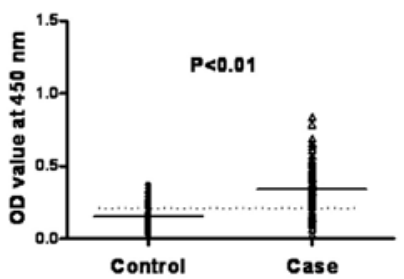

CFP-10 IgG

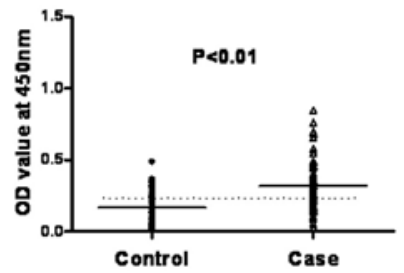

ESPC IgG

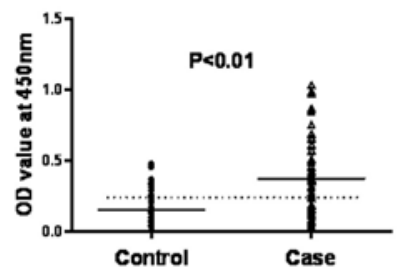

$14 \mathrm{kD} / 38 \mathrm{kD}$ lg G

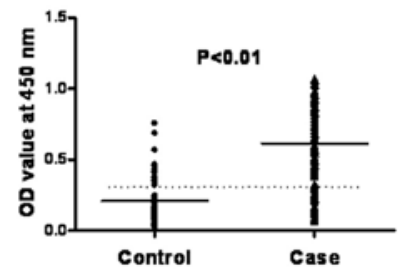

ESAT6/14kD/38kD IgG

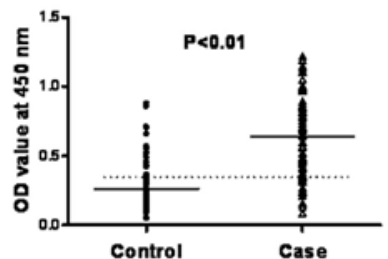

ESAT- 6 IgM

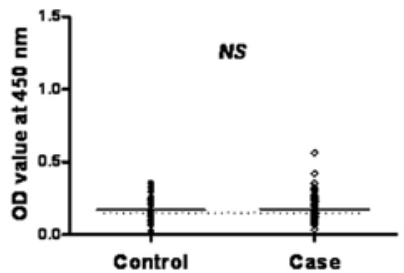

CFP-10 IgM

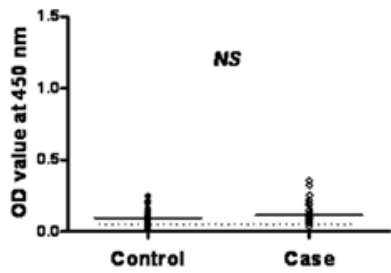

ESPC IgM

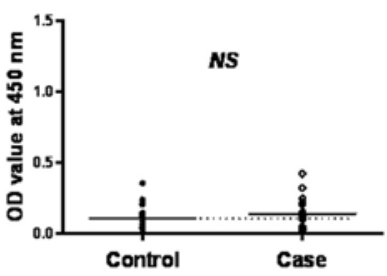

$14 \mathrm{kD} / 38 \mathrm{kD} \operatorname{lgM}$

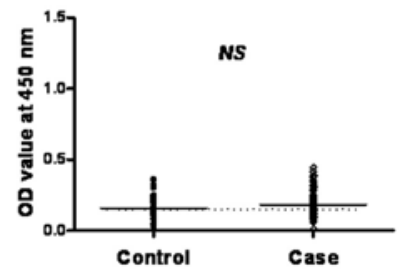

ESAT6/14kD/38kD IgM

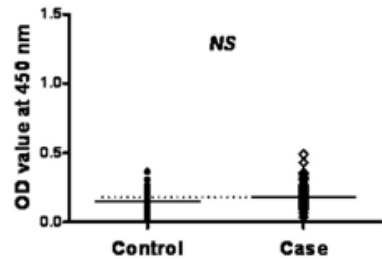

Figure 2. Antibody reactivity to various recombinant MTB antigens with sera derived from active TB patients or healthy control groups. Each scatter represents a tested sample of serum. Data shown are the mean values of individual subjects from three independent experiments; the dotted line denotes the cutoff determined by ROC. Control, the healthy control groups; case, the active pulmonary TB group. P<0.01 denotes statistically significant differences; NS represents no significant difference detected in the assay.

in MTB and M. Bovis isolates but are partially deleted or absent in $M$. bovis BCG as well as in most nontuberculous mycobacteria (NTM) (8). Therefore, these antigens are potential candidate antigens for the serodiagnosis of TB. Previous comparative genomic studies using subtractive DNA hybrid- ization and DNA microarray identified several MTB-specific antigens. In the MTB genome, the region of difference (RD) 1, which encodes several MTB-specific antigens, is present in virulent strains of MTB but is missing from most NTM and M. bovis BCG. The significance of this gene to virulence has 
been demonstrated experimentally; deletion of the $9.5 \mathrm{~Kb}$ RD1 region from $\mathrm{MTB}$ results in attenuation similar to BCG in cultured macrophages and mice (9). The RD1 region has been reported to encode several immunodominant proteins, RV3875, also known as the 6-kDa early secretory antigenic target of MTB (ESAT-6), RV3874 also known as the 10-kDa culture filtrate antigen (CFP-10). ESAT-6 and CFP-10 are both linked with virulent MTB (10). The MTB strains and complements that were positive for ESAT- 6 were virulent, whereas all strains negative for ESAT-6 were avirulent. Similarly, all strains shown to export CFP-10 at high levels were virulent, whereas strains with less CFP-10 in supernatants were avirulent. ESAT-6 and CFP-10 have been reported to be strong targets for humoral and cell-mediated immune response (11). RV3615, also known as ESX-1 substrate protein C (ESPC), is a highly immunodominant RD1-dependent secreted antigen specific for MTB infection (12). Although the ESPC encoding gene is located outside RD1, the expression and secretion of this antigen were RD1-dependent. It has been reported that the ESPC antigen can be detected in the MTB H37RV culture filtrate, but it is absent from the MTB H37RvARD1 culture filtrate. Therefore, ESPC can be recognized specifically in MTB infection but not in BCG-vaccinated persons. ESPC contained multiple $\mathrm{T}$ cell recognize peptides, which have been used for T cell-based immunoassay such as immunospot or cytokine secretion assay. Whether it can be used for antibody detection has yet to be investigated. In this study, we demonstrated that the ESPC antigen also elicits strong humoral immune responses, which can be used for the serodiagnostic assay for MTB infection.

Distinguishing active TB from non TB diseases in clinical practice using ELISA IgG against mycobacterial ESAT-6 and CFP-10 antigen has been proven to be useful. In a study by Kumar et al (11) that was based on the ELISA method, high sensitivity (64.9/66\%) and specificity (88.9/85.2\%) were detected using either ESAT- 6 or CFP-10 antigen. Another report detected $60.4 \%$ sensitivity and $73.8 \%$ specificity using the ESAT-6/CFP-10 recombinant antigen (13). Similar to these previous studies, we found that the sensitivities of ELISA for detecting IgG antibodies against ESAT-6, CFP-10 and ESPC antigen were $69.4,72.5$ and $75.5 \%$, respectively, with specificities of $89.2,78.4$ and $84.3 \%$, respectively. The positive rates in active TB patients were significantly higher than those in healthy controls $(\mathrm{P}<0.01)$.

In addition to the individual MTB antigens, we also generated two recombinant fusion proteins by combined expression of several individual MTB antigens. These included ESAT6/14KD/38KD and 14KD/38KD MTB antigens. The 38-kDa extracellular lipoprotein of MTB is a phosphate-binding protein (PBP) with features very similar to those of the well characterized periplasmic phosphate-binding protein of $E$. coli, which serves as an initial receptor for active transport. It is one of the most important immunogenic antigens of MTB-inducing B- and T-cell responses with high specificity for TB. Using the immunoblot assay, this antigen was the first to be specifically associated with TB infection (14), and it is also considered a prime candidate for the development of new diagnostic reagents for the diagnosis of active TB (15).

The 14KD molecular weight antigen of MTB is also known as $16-\mathrm{kDa}$ antigen, belonging to the $\alpha$ crystalline family of low molecular weight heat shock proteins (HSP) (3). This antigen is one of the prominent antigens of MTB defined by anti TB monoclonal antibodies (MAbs). It was originally identified by three MAbs generated in two separate laboratories and it demonstrated potential as a serodiagnostic target in assay protocols based on MAb competition and direct ELISA (16). This antigen contains B cell epitopes specific for the MTB complex, and it has been suggested that this antigen is immunogenic in the early stages of infection with MTB and in primary TB infection (17).

Compared with the individual ESAT-6, CFP-10 and ESPC antigen, in this study, we noted that the combination expression of the above target antigens ESAT-6/14KD/38KD and $14 \mathrm{KD} / 38 \mathrm{KD}$ can improve the sensitivity at the same time without affecting specificity; 81 and $81.6 \%$ sensitivity for ESAT-6/14KD/38KD and 14KD/38KD antigens was determined in patients with active pulmonary TB. This result indicated that the antibody response against TB infection was heterogeneous (18). Therefore, investigation of the antibody profile as well as the antibody detection against multiple MTB antigens is crucial for improving the detection accuracy of TB.

We also found that the sensitivities and specificities of the IgG test were better than those of the $\operatorname{IgM}$ test; the overall IgM measurement in TB patients had a poor diagnostic sensitivity compared with the healthy control, which is in agreement with previous reports that the sensitivities of the IgM test raised against most of the MTB antigens such as $38 \mathrm{KD}$ antigen, were significantly lower than those of the IgG assays $(19,20)$. Another report showed that IgM measurement using different antigens does not correlate with the presence of TB in children (21).

We consider that this result is likely due to the heterogeneity of the rates of immunochromatographic IgG and IgM antibodies to the target MTB antigens, which depends on the phase of TB infection. In primary pulmonary TB infection, IgM titers were initially high but IgG titers were very low, resulting in low positivity of IgG. In post primary pulmonary $\mathrm{TB}$, low titers of $\operatorname{IgM}$ and high titers of $\mathrm{IgG}$ could explain high rates of positivity of IgG. Healing of post-primary TB was accompanied by substantial lowering of the IgG positivity rate and suppression of IgM positivity (22). We speculated the relatively low titers of IgM antibodies is likely due to the fact that the majority of the patients involved in this study were post-primary pulmonary $\mathrm{TB}$, or, in another case, it may be due to the chronic infection with prolonged exposure to MTB in our setting.

In summary, the present study demonstrated that diagnosis of active TB with a simple multi-antigen ELISA test is feasible. This approach is less expensive and involves minimal technical requirements for testing. Using the recombinant ESAT-6, CFP-10, ESPC, ESAT-6/14KD/38KD, 14KD/38KD antigens, we detected elevated levels of IgG antibody against the target mycobacterial antigens, indicating that this test is of significant value for the rapid diagnosis of MTB infection. A better understanding of the dynamic repertoire of antibody responses in patients with MTB infection as well as other mycobacterial infections may facilitate the development of more sensitive and specific antibody-based methods for the diagnosis of active pulmonary TB infection. Further studies with larger cohorts of patients are required to confirm our results. 


\section{Acknowledgements}

This study was supported by the grants from the Natural Science Foundation of Jiangsu Province China (no. BK2010245) and the National Natural Science Foundation of China (no. 81071298 and 81030013), the grant from National Basic Research Program of China (Grant no. 2009CB918704).

\section{References}

1. WHO: Global Tuberculosis Control. WHO, Geneva, 2011.

2. Ryan K and Ray CG (eds): Sherris Medical Microbiology. 4th edition. McGraw Hill, New York, NY, 2004.

3. Ben-Selma W, Harizi $\mathrm{H}$ and Boukadida J: Immunochromatographic $\operatorname{IgG} / \mathrm{IgM}$ test for rapid diagnosis of active tuberculosis. Clin Vaccine Immunol 18: 2090-2094, 2011.

4. Zhang MM, Zhao JW, Sun ZQ, Liu J, Guo XK, Liu WD and Zhang SL: Identification of RD5-encoded Mycobacterium tuberculosis proteins as B-cell antigens used for serodiagnosis of tuberculosis. Clin Dev Immunol 2012: 738043, 2012.

5. Welch RJ, Lawless KM and Litwin CM: Antituberculosis IgG antibodies as a marker of active Mycobacterium tuberculosis disease. Clin Vaccine Immunol 19: 522-526, 2012

6. Fujita Y, Doi T, Sato K and Yano I: Diverse humoral immune responses and changes in IgG antibody levels against mycobacterial lipid antigens in active tuberculosis. Microbiology 151: 2065-2074, 2005.

7. Imaz MS, Comini MA, Zerbini E, Sequeira MD, Spoletti MJ, Etchart AA, Pagano HJ, Bonifasich E, Diaz N, Claus JD and Singh M: Evaluation of the diagnostic value of measuring $\mathrm{IgG}$, IgM and IgA antibodies to the recombinant 16-kilodalton antigen of Mycobacterium tuberculosis in childhood tuberculosis. Int J Tuberc Lung Dis 5: 1036-1043, 2001.

8. Raja A, Ranganathan UD and Bethunaickan R: Improved diagnosis of pulmonary tuberculosis by detection of antibodies against multiple Mycobacterium tuberculosis antigens. Diagn Microbiol Infect Dis 60: 361-368, 2008

9. Lewis KN, Liao R, Guinn KM, Hickey MJ, Smith S, Behr MA and Sherman DR: Deletion of RD1 from Mycobacterium tuberculosis mimics bacille Calmette-Guérin attenuation. J Infect Dis 187: 117-123, 2003.

10. Hsu T, Hingley-Wilson SM, Chen B, Chen M, Dai AZ, Morin PM, Marks CB, Padiyar J, Goulding C, Gingery M, Eisenberg D, Russell RG, Derrick SC, Collins FM, Morris SL, King CH and Jacobs WR Jr: The primary mechanism of attenuation of bacillus Calmette-Guerin is a loss of secreted lytic function required for invasion of lung interstitial tissue. Proc Natl Acad Sci USA 100: $12420-12425,2003$

11. Kumar G, Dagur P, Singh P, Shankar H, Yadav V, Katoch V, Bajaj B, Gupta R, Sengupta U and Joshi B: Serodiagnostic efficacy of Mycobacterium tuberculosis 30/32-kDa mycolyl transferase complex, ESAT-6, and CFP-10 in patients with active tuberculosis. Arch Immunol Ther Exp 58: 57-65, 2010.
12. Millington KA, Fortune SM, Low J, Garces A, Hingley-Wilson SM, Wickremasinghe M, Kon OM and Lalvani A: Rv3615c is a highly immunodominant RD1 (region of difference 1)-dependent secreted antigen specific for Mycobacterium tuberculosis infection. Proc Natl Acad Sci USA 108: 5730-5735, 2011.

13. Wu X, Yang Y, Zhang J, Li B, Liang Y, Zhang C, Dong M, Cheng $\mathrm{H}$ and $\mathrm{He} \mathrm{J}$ : Humoral immune responses against the Mycobacterium tuberculosis 38-kilodalton, MTB48, and CFP-10/ESAT-6 antigens in tuberculosis. Clin Vaccine Immunol 17: 372-375, 2010.

14. Espitia C, Cervera I, González R and Mancilla R: A 38-kD Mycobacterium tuberculosis antigen associated with infection. Its isolation and serologic evaluation. Clin Exp Immunol 77: 373-377, 1989.

15. Bothamley GH and Rudd RM: Clinical evaluation of a serological assay using a monoclonal antibody (TB72) to the $38 \mathrm{kDa}$ antigen of Mycobacterium tuberculosis. Eur Respir J 7: 240-246, 1994.

16. Verbon A, Hartskeerl RA, Schuitema A, Kolk AH, Young DB and Lathigra R: The 14,000-molecular-weight antigen of Mycobacterium tuberculosis is related to the alpha-crystallin family of low-molecular-weight heat shock proteins. J Bacteriol 174: 1352-1359, 1992.

17. Bothamley GH, Beck JS, Potts RC, Grange JM, Kardjito T and Ivanyi J: Specificity of antibodies and tuberculin response after occupational exposure to tuberculosis. J Infect Dis 166: 182-186, 1992.

18. Lyashchenko K, Colangeli R, Houde M, Al Jahdali H, Menzies D and Gennaro ML: Heterogeneous antibody responses in tuberculosis. Infect Immun 66: 3936-3940, 1998.

19. Demkow U, Filewska M, Białas B, Szturmowicz M, Zielonka T, Wesołowski S, Kuś J, Ziołkowski J, Augustynowicz-Kopeć E, Zwolska Z, Skopińiska-Rábewska E and Rowińska-Zakrzewska E: Antimycobacterial antibody level in pleural, pericardial and cerebrospinal fluid of patients with tuberculosis. Pneumonol Alergol Pol 72: 105-110, 2004.

20. Wilkinson RJ, Hasløv K, Rappuoli R, Giovannoni F, Narayanan PR, Desai CR, Vordermeier HM, Paulsen J, Pasvol G, Ivanyi J and Singh M: Evaluation of the recombinant 38-kilodalton antigen of Mycobacterium tuberculosis as a potential immunodiagnostic reagent. J Clin Microbiol 35: 553-557, 1997.

21. Demkow U, Ziołkowski J, Białas-Chromiec B, Filewska M, Zielonka T, Wasik M and Rowińska-Zakrzewska E: Humoral immune response against mycobacterial antigens in children with tuberculosis. J Physiol Pharmacol 57 (Suppl 4): S63-S73, 2006.

22. Zou YL, Zhang JD, Chen MH, Shi GQ, Prignot J and Cocito C: Serological analysis of pulmonary and extrapulmonary tuberculosis with enzyme-linked immunosorbent assays for anti-A60 immunoglobulins. Clin Infect Dis 19: 1084-1091, 1994. 